\title{
Combinatorial and geometric methods in topology
}

\author{
Carlo Petronio \\ with an appendix by \\ Damian Heard and Ekaterina Pervova
}

October 31, 2018

\begin{abstract}
Starting from the (apparently) elementary problem of deciding how many different topological spaces can be obtained by gluing together in pairs the faces of an octahedron, we will describe the central rôle played by hyperbolic geometry within three-dimensional topology. We will also point out the striking difference with the two-dimensional case, and we will review some of the results of the combinatorial and computational approach to three-manifolds developed by different mathematicians over the last several years. MSC (2000): 57M50 (primary), 57M25 (secondary).
\end{abstract}

The octahedron, denoted henceforth by $O$, is one of the favourite toys of every geometer, being one of the five Platonic solids. In this note we will investigate the following:

Question 0.1. How many different topological spaces can be obtained by gluing together in pairs the faces of $O$ ?

Question 0.1 has a rather transparent combinatorial flavour and appears to be well-suited to computer investigation, but the complete answer would be extremely difficult to obtain without the aid of some rather sophisticated geometric tools developed over the last three decades by a number of mathematicians. It is indeed mostly thanks to hyperbolic geometry that one is 
able to show that certain gluings of $O$, despite being very similar to each other under many respects, are in fact distinct.

There is a very natural family of lesser siblings of Question 0.1, involving (two-dimensional) polygons rather than a (three-dimensional) polyhedron (as the octahedron is), and we will show below that the answers to these lower-dimensional questions are sharply different from both a qualitative and quantitative viewpoint. More precisely, it turns out that identifying the spaces obtained by gluing together the edges of a polygon is very easy, and that the number of possible different results is very small if compared to the number of (combinatorially inequivalent) gluing patterns. On the other hand, in dimension three identifying the results is often only possible using hyperbolic geometry, and there is a rather large variety of different results. This can be viewed as a manifestation of the crucial rôle played by hyperbolic geometry in the context of three-dimensional topology, as chiefly witnessed by Thurston's geometrization.

As opposed to looking at lower-dimensional analogues of Question 0.1, one can also view it as a special instance of a more general family of threedimensional problems, where one considers larger polyhedra (or finite families of polyhedra). These problems have attracted a considerable attention during the last several years, and we will include a brief survey of the main results obtained.

ACKnowledgements. Part of this work was carried out while the author was visiting the Université Paul Sabatier in Toulose and the Columbia University in New York. He is grateful to both these institutions for financial support, and he would like to thank Michel Boileau and Dylan Thurston for their warm hospitality and inspiring mathematical discussions. He is also glad that Damian Heard and Katya Pervova accepted to contribute to the paper with an appendix where they explain how they have determined some of the entries in Table 7 .

\section{One dimension down}

Let us denote by $P_{k}$ a polygon with $2 k$ edges. Since we are only interested in topology, this makes sense for $k \geqslant 1$. In other words, $P_{k}$ is just the 2 -dimensional closed disc with boundary circle subdivided into $2 k$ segments. 
Question 1.1. How many different topological spaces can be obtained by gluing together in pairs the edges of $P_{k}$ ?

We will investigate this question only with a restriction on the gluings. Namely, we endow $P_{k}$ with some orientation, we give its edges the induced orientation, and we require the gluing maps to reverse this orientation of the edges. With this proviso a gluing pattern is determined by the instructions of which edges should get glued to which. If one gives labels from 1 to $2 k$ to the edges of $P_{k}$ (as one should do if one wants to feed $P_{k}$ to a computer) one easily sees that the number of different patterns is $(2 k-1)$ !! This is because one must first choose which edge gets glued to edge number 1 , and there are $2 k-1$ choices for this. Then one must locate the first edge which is left free after the first gluing, and select one of the remaining $2 k-3$ edges to glue it to, and so on.

As just mentioned, counting the different gluing patterns is easy, but one must also note that $P_{k}$ has some symmetries, given by the action of the dihedral group $D_{2 k}$, which has $4 k$ elements. So the number of combinatorially inequivalent patterns is smaller than $(2 k-1)$ !!, but at least $(2 k-1) ! ! / 4 k$ (and actually always strictly larger than this value, because some patterns will have symmetries themselves). Giving a general formula for the number of inequivalent patterns for arbitrary $k$ is probably rather complicated, but it is not too difficult to write a piece of computer code that does the counting for small $k$. The author has implemented this counting algorithm using the language Haskell and computed the number of gluings for $k \leqslant 6$, with results as illustrated below.

Knowing how many gluing patterns exist for some $k$, however, does not answer Question 1.1, because inequivalent patterns may well give homeomorphic spaces after gluing, and as a matter of fact they very often do. But one can notice that the topological space resulting from a gluing is always a surface, and an orientable one under our restriction that the gluings should reverse the orientation of the edges. Now, an orientable surface $\Sigma$ is classified by its genus $g(\Sigma)$, an integer that can attain any non-negative value, and the genus can easily be computed from the Euler characteristic thanks to the relation $\chi(\Sigma)=2(1-g(\Sigma))$. Computing $\chi(\Sigma)$ for a surface $\Sigma$ obtained by gluing together the edges of $P_{k}$ is also very easy, because $\chi(\Sigma)=v-k+1$, where $v$ is the number of equivalence classes of vertices under the relation generated by the gluings. This implies that $\chi(\Sigma) \geqslant 2-k$, so $g(\Sigma) \leqslant k / 2$. On the other hand it is well known that the surface of genus $g>0$ can be re- 


\begin{tabular}{c||c|c|c|c|c|c}
$k$ & 1 & 2 & 3 & 4 & 5 & 6 \\
\hline \hline$\#$ (gluings) & 1 & 3 & 15 & 105 & 945 & 10395 \\
\hline$\#$ (inequivalent gluings) & 1 & 2 & 5 & 17 & 79 & 554 \\
\hline$\#$ (resulting surfaces) & 1 & 2 & 2 & 3 & 3 & 4
\end{tabular}

Table 1: Numbers of gluing patterns for the $2 k$-edged polygon and of the resulting surfaces

alized by gluing the edges of $P_{2 g}$, and as a matter of fact also all lower-genus surfaces can. This discussion implies the following:

Proposition 1.2. The number of combinatorially inequivalent patterns for the edges of $P_{k}$ is at least $(2 k-1) ! ! / 4 k$, while the number of distinct resulting surfaces is $[k / 2]+1$.

The exact figures for $k \leqslant 6$ are given in Table 1. The qualitative conclusion we can draw from the above discussion, and in particular from Proposition 1.2 and Table 1, is that in two dimensions it is easy to recognize the surface resulting from a gluing of the edges of a polygon, and the number of surfaces that arise is very small compared to the number of inequivalent gluings. We will see that the situation is sharply different in three dimensions.

\section{Three-manifolds (with boundary)}

In the previous section we have taken for granted that the result of a gluing of the edges of a polygon is a surface, namely a (compact) topological space locally homeomorphic to the plane $\mathbb{R}^{2}$. Since we will see that a similar result almost but not quite holds in three dimensions, we will now prove the 2dimensional statement, in order to show where the difficulty can arise. As we do this we momentarily drop the restriction that the gluing should reverse the orientation of the edges, because the conclusion holds in general.

Proposition 2.1. The space resulting from an arbitrary simplicial pairing of the edges of the polygon $P_{k}$ is a surface.

Proof. Denote by $X$ the result of the gluing and by $\pi: P_{k} \rightarrow X$ the projection. There are three different sorts of points $x$ of $X$ that we must look at, depending on what $\pi^{-1}(x)$ is. The possibilities are as follows: 
- $\pi^{-1}(x)$ is a single interior point $p$ of $P_{k}$;

- $\pi^{-1}(x)$ consists of two points $p_{1}$ and $p_{2}$ lying in the interior of two edges of $P_{k}$;

- $\pi^{-1}(x)$ consists of some vertices $v_{1}, \ldots, v_{n}$ of $P_{k}$.

For the first type, since $\pi$ is a local homeomorphism near $p$, it is clear that $x$ is a surface point of $X$. For the second type, a neighbourhood of $x$ in $X$ is obtained by gluing neighbourhoods of $p_{1}$ and $p_{2}$ in $P_{k}$. These neighbourhoods can be chosen to be half-discs and the gluing identifies their diameters, so again $x$ is a surface point. Getting to the last type of points, we note that a neighbourhood of each $v_{i}$ in $P_{k}$ can be viewed as the cone with vertex $v_{i}$ over a segment. Therefore a neighbourhood of $x$ in $X$ is the cone over the space obtained from the union of $n$ segments by gluing together in pairs their endpoints. This space is connected, so it is a circle, whence a neighbourhood of $x$ in $X$ is a disc, which proves that $x$ is again a surface point.

Turning to three dimensions and our octahedron $O$, we introduce again the restriction that the gluings of the faces should reverse the orientation induced by some orientation of $O$. For instance, if we label by $v_{0}, \ldots, v_{5}$ the vertices of $O$ so that the faces are $\left(v_{i}, v_{j}, v_{j+1}\right)$ for $i=0,5$ and $j=1, \ldots, 4$, with the convention that $j+1=1$ for $j=4$, then the maps

$$
\left(v_{0}, v_{1}, v_{2}\right) \longrightarrow\left(v_{0}, v_{3}, v_{2}\right), \quad\left(v_{0}, v_{1}, v_{2}\right) \longrightarrow\left(v_{5}, v_{1}, v_{2}\right)
$$

define gluings that are acceptable for us, while

$$
\left(v_{0}, v_{1}, v_{2}\right) \longrightarrow\left(v_{0}, v_{2}, v_{3}\right), \quad\left(v_{0}, v_{1}, v_{2}\right) \longrightarrow\left(v_{5}, v_{2}, v_{1}\right)
$$

do not. We also recall that a 3 -manifold is a space locally homeomorphic to $\mathbb{R}^{3}$, and we denote by $O^{(0)}$ the set of vertices of $O$.

Proposition 2.2. Let $\varphi$ be a simplicial orientation-reversing pairwise gluing of the faces of the octahedron $O$. Define

$$
X(\varphi)=O / \varphi, \quad V(\varphi)=O^{(0)} / \varphi, \quad Y(\varphi)=X(\varphi) \backslash V(\varphi) .
$$

Then $Y(\varphi)$ is a 3-manifold. 
Proof. Let $\pi: O \rightarrow X(\varphi)$ be the projection. We first prove the following claim: the restriction of $\pi$ to the interior of each edge of $O$ is injective. To show this, we assume $\pi$ identifies together some edges $e_{0}, \ldots, e_{m-1}$ of $O$. More precisely, we assume $e_{i}$ is contained in some faces $F_{i}$ and $F_{i}^{\prime}$ of $O$ and that there is a gluing from $F_{i}^{\prime}$ to $F_{i+1}$ mapping $e_{i}$ to $e_{i+1}$, with indices understood modulo $m$. Furthermore we give to each $F_{i}$ and $F_{i}^{\prime}$ the orientation induced by $O$, and to each $e_{i}$ the orientation induced by $F_{i}$. Since $F_{i}^{\prime}$ induces on $e_{i}$ the negative orientation and the gluing from $F_{i}^{\prime}$ to $F_{i+1}$ reverses the orientation, we see that this gluing matches the orientations of $e_{i}$ and $e_{i+1}$. Therefore the composition of all the gluings $e_{0} \rightarrow e_{1} \rightarrow \ldots \rightarrow e_{m-1} \rightarrow e_{0}$ is an orientation-preserving simplicial self-gluing of $e_{0}$. This gluing is the identity, so $\pi$ restricted to the interior of $e_{0}$ is injective. Our claim is proved.

As in the 2-dimensional case we now take $x \in Y(\varphi)$ and distinguish according to what $\pi^{-1}(x)$ is. The possibilities are as follows:

- $\pi^{-1}(x)$ is a single interior point of $O$;

- $\pi^{-1}(x)$ consists of two interior points of some faces of $O$;

- $\pi^{-1}(x)$ consists of some points $p_{1}, \ldots, p_{n}$ lying in the interior of $n$ edges of $O$.

Again points of the first two types are obviously 3-manifold points. For the last type we note that a neighbourhood of $p_{i}$ in $O$ can be viewed as the cone with vertex $p_{i}$ over the bigon $P_{2}$. Therefore a neighbourhood of $x$ in $X(\varphi)$ is the cone over a connected space obtained from a finite union of bigons by gluing together in pairs their edges. Of course by performing all but one gluing we can reduce to the case of a single bigon. Now, depending on whether the gluing reverses or preserves the orientation induced on the edges of the bigon, the resulting space is either the 2-sphere or a non-orientable surface, called the projective plane. In the first case, since the cone over a 2 -sphere is a 3 -disc, we see that $x$ is a 3 -manifold point. The same conclusion would be false in the second case, but an argument very close to that presented above to establish the claim shows that the second case actually never occurs under the current restriction that the gluings should reverse the orientation. The proof is complete.

We will now see that it is not possible to improve Proposition 2.2 showing that the whole of $X(\varphi)$ is a 3-manifold. (We will later see that this is rather an advantage than a disadvantage). To this end we define the truncated 
octahedron $O_{\mathrm{t}}$ as $O$ minus small open pyramidal neighbourhoods of its vertices, chosen so that any simplicial gluing of the faces of $O$ will match their triangular faces. Note that $O_{\mathrm{t}}$ is a polyhedron bounded by 8 hexagons and 6 squares, and that any simplicial gluing of the faces of $O$ induces a gluing between the hexagonal faces of $O_{\mathrm{t}}$. Before stating our result we recall that a 3-manifold with boundary is a space $M$ locally homeomorphic to a closed half-space of $\mathbb{R}^{3}$. The boundary $\partial M$ of $M$ consists of the points not having neighbourhoods homeomorphic to $\mathbb{R}^{3}$, and it is a (possibly disconnected) surface.

Proposition 2.3. Fix the notation of Proposition 2.2 and let $M(\varphi)$ be the space obtained from the truncated octahedron $O_{\mathrm{t}}$ by the gluing induced from $\varphi$. Then $M(\varphi)$ is a 3-manifold with boundary. Moreover:

- There is a natural correspondence between the components of $\partial M(\varphi)$ and the elements of $V(\varphi)$;

- A point of $V(\varphi)$ is a 3-manifold point of $X(\varphi)$ if and only if the corresponding component of $\partial M(\varphi)$ is a sphere.

Proof. If we remove from $O_{\mathrm{t}}$ its 6 square faces, we get a space homeomorphic to $O \backslash O^{(0)}$. This easily implies that the space $\operatorname{Int}(M(\varphi))$ obtained from $M(\varphi)$ by removing the projections of the squares is homeomorphic to $Y(\varphi)$, so it is a 3-manifold without boundary. The complement in $M(\varphi)$ of $\operatorname{Int}(M(\varphi))$ is obtained from the squares by gluing together in pairs their edges, so it is a surface $\partial M(\varphi)$. It is now immediate to check that $\operatorname{Int}(M(\varphi))$ and $\partial M(\varphi)$ fit together nicely to give $M(\varphi)$ the structure of a 3-manifold with boundary.

By the very construction, a neighbourhood in $X(\varphi)$ of an element of $V(\varphi)$ is the cone over a component of $\partial M(\varphi)$. The conclusion easily follows from the remark that the cone over a surface is a 3-manifold if and only if the surface is the sphere.

\section{Partial answers from lower dimension}

To face Question 0.1 the first issue is of course to determine how many gluing patterns can be chosen for the faces of the octahedron. The situation is almost but not quite similar to the 2-dimensional case of $P_{k}$. On one hand, to determine a gluing, we must again start by choosing which faces get glued to which, and this gives a wealth of $(8-1) ! !=105$ choices. But on the 
other hand this first information, which is sufficient for $P_{k}$ to determine the gluing, is not sufficient for $O$, because there are three distinct orientationreversing simplicial homeomorphisms between two given faces (obtained from each other by pre- or post-composition with a rotation). The total number of gluing patterns is therefore $105 \cdot 3^{4}=8505$.

Since combinatorially equivalent gluing patterns of course give rise to homeomorphic glued spaces, the next task is to let the symmetry group of the octahedron act on the 8505 gluings. The group has 48 elements but several gluing patterns have themselves non-trivial symmetries, so it turns out that the actual number (that we have determined by computer, again using some Haskell code) is significantly larger than 8505/48 = 177.1875:

Proposition 3.1. There exist 298 combinatorially inequivalent patterns of orientation-reversing gluings of the faces of $O$.

Having carried out this preliminary combinatorial work, to attack Question 0.1 we now need to start facing topological issues. But the discussion of the previous section suggests that, from the point of view of the topology of 3-manifolds, the following is a more natural problem than the original one:

Question 3.2. How many different 3-manifolds with boundary $M(\varphi)$ can be obtained from the truncated octahedron $O_{\mathrm{t}}$ under a gluing of its hexagonal faces induced by some simplicial orientation-reversing gluing $\varphi$ of the faces of the octahedron $O$ ?

Two homeomorphic 3-manifolds of course have homeomorphic boundaries, and we have seen above that identifying the topological type of a surface is easy, so the first natural thing to do to attack this question is to subdivide the inequivalent gluings $\varphi$ according to the type of $\partial M(\varphi)$. The figures (obtained by computer) are given in Table 2, where $S$ is the sphere, $T$ is the torus and $\Sigma_{g}$ is the surface of genus $g \geqslant 2$. (Of course we could have denoted $S$ and $T$ by $\Sigma_{0}$ and $\Sigma_{1}$ respectively. However we already know from Proposition 2.3 that $S$ has a totally peculiar status among surfaces from the viewpoint of topology, whereas we will see in Section 4 that $T$ is peculiar from the viewpoint of hyperbolic geometry. So we prefer to keep the notation clearly distinct).

Table 2 shows that the type of $\partial M(\varphi)$ gives rather limited information towards the answer to Question 3.2. In addition this question is indeed not equivalent to our original one, but a small variation on it turns out to be 


\begin{tabular}{c|c}
$\partial M(\varphi)$ & \#(inequivalent $\varphi^{\prime}$ s) \\
\hline \hline$S$ & 23 \\
\hline$S \sqcup S$ & 8 \\
\hline$S \sqcup S \sqcup S$ & 4 \\
\hline$S \sqcup S \sqcup S \sqcup S$ & 1 \\
\hline$S \sqcup S \sqcup S \sqcup S \sqcup S$ & 1 \\
\hline$T$ & 67 \\
\hline$T \sqcup S$ & 10 \\
\hline$T \sqcup S \sqcup S$ & 4 \\
\hline$T \sqcup T$ & 8 \\
\hline$T \sqcup T \sqcup S$ & 1 \\
\hline$\Sigma_{2}$ & 113 \\
\hline$\Sigma_{2} \sqcup T$ & 2 \\
\hline$\Sigma_{3}$ & 56 \\
\hline \hline Total & 298
\end{tabular}

Table 2: Numbers of inequivalent gluings $\varphi$ of the faces of $O$, subdivided according to the topological type of $\partial M(\varphi)$

so. This will follow from the next easy result, to state which we introduce a little extra notation. Given a 3-manifold $M$ we will denote by $\widehat{M}$ the space obtained from $M$ by attaching a 3-disc to each component of $\partial M$ homeomorphic to the sphere $S$. Note that $\widehat{M}$ is again a 3 -manifold with boundary, but if $\partial M$ consists of spheres only then $\partial \widehat{M}$ is empty, i.e. $\widehat{M}$ is a closed 3-manifold.

Proposition 3.3. $X(\varphi)$ and $X\left(\varphi^{\prime}\right)$ are homeomorphic to each other if and only if $\widehat{M}(\varphi)$ and $\widehat{M}\left(\varphi^{\prime}\right)$ are homeomorphic to each other.

In the rest of the paper we will consider the following reformulation of Question 0.1

Question 3.4. How many different 3-manifolds $\widehat{M}(\varphi)$ exist as $\varphi$ varies among the simplicial orientation-reversing gluings of the faces of the octahedron $O$ ?

The contribution of 2-dimensional topology towards the answer to this question is easily deduced from Table 2 and summarized in Table 3 , 


\begin{tabular}{c|c}
$\partial \widehat{M}(\varphi)$ & $\#$ (inequivalent $\varphi$ 's) \\
\hline \hline$\emptyset$ & 37 \\
\hline$T$ & 81 \\
\hline$T \sqcup T$ & 9 \\
\hline$\Sigma_{2}$ & 113 \\
\hline$\Sigma_{2} \sqcup T$ & 2 \\
\hline$\Sigma_{3}$ & 56 \\
\hline \hline Total & 298
\end{tabular}

Table 3: Numbers of inequivalent gluings $\varphi$ of the faces of $O$, subdivided according to the topological type of $\partial \widehat{M}(\varphi)$

\section{The magic of hyperbolic geometry in three dimensions}

According to Table 3, the answer to Question 3.4, and hence to Question 0.1 , could be any number between 6 and 298, so the information provided by the boundary is very weak. To go further we will turn from purely combinatorial and topological methods to geometric ones. To this end we recall that a Riemannian metric [1] on a manifold $M$ (possibly with non-empty boundary) is a smoothly varying inner product on the tangent spaces at the points of $M$. Given such a metric on $M$ one can:

- Define the length $L(\alpha)$ of a smooth path $\alpha$ in $M$ by integrating the norm of the tangent vector $\dot{\alpha}$;

- Introduce on $M$ the distance $d$ between any two points, given by the infimum of the lengths of smooth paths joining them;

- Define a geodesic as a smooth path locally realizing the distance between the points it contains;

- Define a submanifold $N$ of $M$ (for instance, the boundary $\partial M$ ) to be totally geodesic if any geodesic meeting $N$ in more than one point is actually contained in $N$; for the sake of brevity we will henceforce omit the word "totally"; 
- Define the (possibly infinite) volume of $M$ by integrating the square root of the determinant of the matrix representing the metric in local charts;

- Assign to each 2-plane $P$ contained in a tangent space $T_{x} M$ a real number $K_{x}(P)$ called the sectional curvature of $M$ at $x$ along $P$.

We recall that for a Riemannian 2-manifold, i.e. a surface, the curvature at a point $x$ measures the extent to which the circle of a very small radius $r$ centred at $x$ fails to have length $2 \pi r$, according to the formula

$$
K_{x}=\lim _{r \rightarrow 0^{+}} 6 \cdot \frac{2 \pi r-L\left(C_{x}(r)\right)}{r^{2}}, \quad C_{x}(r)=\{y: d(x, y)=r\} .
$$

(The 6 is introduced in the limit so that the round sphere of radius 1 in $\mathbb{R}^{3}$ with metric induced from $\mathbb{R}^{3}$ has curvature +1 ). The sectional curvature $K_{x}(P)$ is the curvature at $x$ of a germ of geodesic surface with tangent plane $P$ at $x$.

We will now restrict to the type of metrics that turns out to be mostly relevant for 3-dimensional topology. To this end we consider an orientable manifold $M$ with compact boundary $\partial M$ (but possibly non-compact itself). We define a Riemannian metric on $M$ to be hyperbolic if:

- The sectional curvature is -1 at every point and along every tangent plane;

- $M$ is a complete metric space with respect to the distance induced by the Riemannian metric;

- The volume of $M$ is finite.

A number of spectacular results have been proved about hyperbolic manifolds in dimension 3 over the last 30 years, many of which due to or inspired by Bill Thurston [27]. We list here those we will need to refer to, but we mention that there are very many more:

- Mostow's rigidity theorem [27, 24, 2]: Any two hyperbolic metrics on the same manifold are isometric to each other. This implies in particular that any hyperbolic invariant, such as the volume or the length of the shortest geodesic, is automatically a topological invariant, 
which gives an extremely powerful method for distinguishing manifolds. Note that the assumption that the volume should be finite is crucial for rigidity;

- Cusps and compactification [27, 24, 2]: If $M$ is hyperbolic then $M$ decomposes as $M_{0} \cup C_{1} \cup \ldots \cup C_{k}$, where $M_{0}$ is a compact manifold with boundary $\partial M_{0}$ consisting of $\partial M$ together with $k$ tori $T_{1}, \ldots, T_{k}$, and $C_{i}=T_{i} \times[0, \infty)$ with $T_{i}=T_{i} \times\{0\}$. In particular, $M$ compactifies to a manifold $\bar{M}$ by adding the tori $T_{i} \times\{\infty\}$. Since each boundary component of $M$ has a metric of constant curvature -1, the GaussBonnet formula [1] implies that it cannot be the sphere or the torus. So $\bar{M}$ is a compact manifold without boundary spheres and $M$ is obtained from $\bar{M}$ by removing all the boundary tori;

- Thurston's hyperbolization proved by Perelman [27, 21, 22, 23]: If $\bar{M}$ is a compact orientable manifold without boundary spheres and different from the solid torus, $\pi_{1}(M)$ is an infinite group, and $M$ does not contain any essential embedded sphere, disc, annulus or immersed torus, then the manifold $M=\bar{M} \backslash$ (boundary tori) is hyperbolic. The notion of "essentiality" of an embedded surface is too technical to be reproduced here, but it basically means that cutting $\bar{M}$ along the surface one gets some non-obvious simplification of $\bar{M}$; an immersed torus is essential if its fundamental group injects in that of $\bar{M}$ but none of its conjugates is contained in a subgroup of $\pi_{1}(M)$ arising from a toric boundary component. Combining two well-known general theorems (the Haken-Kneser-Milnor decomposition along spheres [10] and the Jaco-Shalen-Johansson decomposition along tori [18]) one knows that a set of "building blocks" for all 3-manifolds is given by:

- those as described in the statement of the hyperbolization theorem;

- those finitely covered by a closed simply connected manifold;

- those of a type that has been classified long ago (Seifert manifolds).

So Thurston's hyperbolization theorem together with the positive solution to the Poincaré conjecture (also proved by Perelman) can be interpreted as a classification result for all 3-manifolds, and shows that hyperbolic geometry plays a central rôle in 3-dimensional topology; 
- Epstein-Penner canonical decomposition [4]: If $M$ is hyperbolic, $\partial M=\emptyset$ and $M$ is non-compact then $M$ has a canonical realization as a gluing of ideal polyhedra. An ideal polyhedron is just one with its vertices removed, and we have already met in Proposition 2.2 the idea of gluing together such objects. The statement means that the geometry (and hence the topology, thanks to rigidity) of $M$ determines not only the number of polyhedra and their type but also the combinatorics of the gluing pattern;

- Kojima canonical decomposition [11, 12]: If $M$ is hyperbolic and $\partial M \neq \emptyset$ then $M$ has a canonical realization as a gluing of truncated/ideal polyhedra. This statement has precisely the same meaning as the previous one, except that in this case some vertices of the polyhedra are removed and give rise to the cusps of $M$, while other vertices are truncated, and the truncation polygons glue together to give the geodesic boundary of $M$;

\section{- Algorithmic approach:}

- Search for hyperbolic structure [27, 30, 2, 29, 8, 9]: Given a manifold $\bar{M}$ realized as a gluing of truncated tetrahedra, one can try to build the hyperbolic structure on $M=\bar{M} \backslash$ (boundary tori) by choosing for the tetrahedra geometric shapes (parameterized by numbers) and imposing these structures to glue together nicely (which translates into equations in the parameters). This searching method for the hyperbolic structure is not guaranteed to always work, but in practice it most often does if one starts from a realization having the minimal possible number of tetrahedra. The algorithm was implemented in the case of empty boundary by Weeks and in the case of non-empty boundary by FrigerioMartelli-Petronio and by Heard;

- Search for Epstein-Penner decomposition [25, 30]: The canonical realization of a cusped hyperbolic manifold with empty boundary can be searched for algorithmically starting from a geometric realization as a gluing of ideal tetrahedra. This method is based on a certain "tilt formula" due to Sakuma and Weeks. Again the algorithm is not guaranteed to always converge but in practice it does. It was implemented by Weeks; 
- Search for Kojima decomposition [29, 8, 9]: The canonical realization of a cusped hyperbolic manifold with non-empty boundary can be searched for algorithmically starting from a geometric realization as a gluing of truncated/ideal tetrahedra. This method is based on a variation of the "tilt formula" established by Ushijima. Once more the algorithm is not guaranteed to always converge but in practice it does; it was implemented by Frigerio-MartelliPetronio and by Heard.

\section{Answers from hyperbolic geometry}

In this section we will employ the rich technology of hyperbolic geometry to show that the combinatorially inequivalent gluing patterns of Table 3actually give rise to very many different manifolds. We begin with the following striking fact, that was initially discovered from a computer experiment [7] and was later proved theoretically:

Proposition 5.1. The 56 gluing patterns of Table 3 giving rise to a boundary of genus 3 define 56 pairwise distinct hyperbolic manifolds with geodesic boundary. These manifolds all have the same volume $11.448776 \ldots$. The homology is $\mathbb{Z}^{3}$ for 52 of them and $\mathbb{Z}_{3} \times \mathbb{Z}^{3}$ for four of them.

Proof. An easy computation of Euler characteristic shows that a gluing $\varphi$ defines a manifold $\widehat{M}(\varphi)$ bounded by $\Sigma_{3}$ if and only if it identifies all 12 edges to each other. We want to show that such an $\widehat{M}(\varphi)$ is hyperbolic with geodesic boundary by choosing a hyperbolic shape of the truncated octahedron $O_{\mathrm{t}}$ that is matched by $\varphi$. Since all edges are glued together, this can only happen if the geometric shape is such that all edges have the same length, i.e. $O_{\mathrm{t}}$ is regular. If this is the case, all dihedral angles are also the same, so they must all be $2 \pi / 12$. Such an octahedron certainly does not exist in Euclidean or spherical geometry, but it does in hyperbolic geometry. This implies that $\widehat{M}(\varphi)$ is indeed hyperbolic.

Let us now analyze the Kojima canonical decomposition of $\widehat{M}(\varphi)$. To this end we recall [11, 12] that it is dual to the cut locus of the boundary, i.e. to the set of points having multiple shortest paths to $\partial \widehat{M}(\varphi)$. Using the fact that $\widehat{M}(\varphi)$ is the gluing of a regular truncated octahedron, which is totally symmetric, it is not too difficult to show that the Kojima decomposition is given by the octahedron itself, with its gluing pattern $\varphi$. This implies 
that the geometry of $\widehat{M}(\varphi)$, and hence its topology, determines $\varphi$. Therefore different $\varphi$ 's give rise to different $\widehat{M}(\varphi)$ 's.

The fact that all the $\widehat{M}(\varphi)$ 's have the same volume is obvious. For the computation of the value of this volume and for the determination of the homology we address the reader to [7].

Turning to the other gluing patterns in Table 3 , we now cite some facts that follow from the results in [7]:

Proposition 5.2. The two $\varphi$ 's such that $\partial \widehat{M}(\varphi)=\Sigma_{2} \sqcup T$ define distinct hyperbolic manifolds with geodesic boundary and one cusp. The group of 113 inequivalent $\varphi$ 's such that $\partial \widehat{M}(\varphi)=\Sigma_{2}$ contains:

- A group of 14 's defining 14 distinct hyperbolic manifolds with geodesic boundary and Kojima decomposition given by a truncated octahedron;

- A group of 8 's defining 8 distinct hyperbolic manifolds with geodesic boundary and Kojima decomposition given again by a truncated octahedron, but with a different geometric shape;

- A group of 4 's defining 4 distinct hyperbolic manifolds with geodesic boundary and Kojima decomposition given by two square pyramids.

The manifolds arising from these three groups of gluings are all distinct from each other. Moreover any other hyperbolic $\widehat{M}(\varphi)$ with $\partial \widehat{M}(\varphi)=\Sigma_{2}$ has Kojima decomposition consisting of tetrahedra only.

The gluing patterns in Table 3 not covered by the results just stated have been analyzed by Damian Heard using his software "Orb", with the following results:

Proposition 5.3. - Consider the set of 87 inequivalent $\varphi$ 's such that $\partial \widehat{M}(\varphi)=\Sigma_{2}$ but not included in Proposition 5.2. Then this set contains at least 37 elements defining hyperbolic manifolds with geodesic boundary, and these manifolds are all distinct from each other;

- The set of 81 inequivalent $\varphi$ 's with $\partial \widehat{M}(\varphi)=T$ contains at least 11 elements defining one-cusped hyperbolic manifolds, and there are 9 different manifolds arising from these $11 \varphi$ 's; 
- The set of 9 inequivalent $\varphi$ 's with $\partial \widehat{M}(\varphi)=T \sqcup T$ contains at least 2 elements defining two-cusped hyperbolic manifolds, and these two manifolds are distinct.

As one sees, hyperbolic geometry only gives a partial answer to Question 3.4 when the boundary is not $\Sigma_{3}$. In addition the octahedron is often not related to the geometry of the result. For this reason we will consider a more general question in the next section, but the appendix contains the full answer to the original question on the gluings of the octahedron.

\section{Other enumeration problems and results}

Since the octahedron can be subdivided (in three different ways) into four tetrahedra, listing the different manifolds arising from gluings of the octahedron is a special case of the following:

Question 6.1. Given $c>0$, how many different 3-manifolds can be obtained by gluing together in pairs the faces of a disjoint union of $c$ tetrahedra?

As usual we consider this question only under the restriction that the face-pairings should be orientation-reversing, and we remove from the space obtained after the gluing an open regular neighbourhood of the non-manifold points, so to get compact manifolds bounded by surfaces of positive genus.

A systematic approach to Question 6.1 was proposed by Matveev [17, who introduced a certain complexity theory. To describe it, we need to recall the following definitions and facts [10]:

- A 3-manifold is called prime if every embedded 2-sphere it contains is the boundary of an embedded 3-disc;

- The connected sum \# of two 3-manifolds is obtained by removing an open 3-disc from each and gluing together the boundary spheres thus created;

- Every 3-manifold can be expressed in a unique way as a connected sum of a finite number of prime 3-manifolds.

With this in mind, Matveev noticed that a good notion of complexity for a 3-manifold $M$, i.e. a measure $c(M) \in \mathbb{N}$ of how complicated $M$ is, should have the property of being additive under connected sum. This is however 
certainly not the case if one defines $c(M)$ as the minimal number of tetrahedra needed to realize $M$ via face-pairings, because $S^{3}$ would have positive complexity, but $c\left(M \# S^{3}\right)=M$ for any $M$. This problem was solved in [17] by defining $c(M)$ through a different and more flexible object than a decomposition into tetrahedra, called a simple spine. We will not recall what such a thing is in this paper, but we will mention that among simple spines there are some having additional properties, called special spines, that turn out to be perfectly equivalent to decompositions into tetrahedra. The main features of complexity theory, that we state for the case of closed orientable manifolds but have extensions to more general situations, are now the following:

- $c(M)$ is a non-negative integer;

- $c\left(M_{1} \# M_{2}\right)=c\left(M_{1}\right)+c\left(M_{2}\right)$;

- The prime 3-manifolds $M$ with $c(M)=0$ are the sphere $S^{3}$, the projective space $\mathbb{R P}^{3}$, the lens space $L(3,1)$, and the product $S^{2} \times S^{1}$;

- If $M$ is prime and $c(M)>0$ then $c(M)$ is precisely the minimal number of tetrahedra needed to realize $M$ via face-pairing.

¿From the viewpoint of complexity theory, the following variation on Question 6.1 is therefore more natural:

Question 6.2. Given $c>0$, how many different prime 3-manifolds can be obtained by gluing together in pairs the faces of a disjoint union of $c$ tetrahedra?

This question was investigated both theoretically and using computers by Matveev, Martelli-Petronio, Martelli and Matveev (see [13] for a list of the relevant papers and websites). The answer was obtained for $c \leqslant 12$ and it is described in Table 4, where the information of how many hyperbolic manifolds were found is also added. We further note that the first four such manifolds, appearing in complexity 9 and first discovered in [14], are precisely those with the four smallest known volumes. This supports a conjecture of Matveev and Fomenko that low volume should appear in low complexity.

We have spoken so far of closed manifolds, but a variation of complexity theory applies to bounded compact 3-manifolds too. However in this case the equality between $c(M)$ and the minimal number of tetrahedra in a decomposition requires $M$ not only to be prime but also to have incompressible 


\begin{tabular}{c||c|c|c|c|c|c|c|c|c|c|c|c|c}
$c$ & 0 & 1 & 2 & 3 & 4 & 5 & 6 & 7 & 8 & 9 & 10 & 11 & 12 \\
\hline \hline tot & 4 & 2 & 4 & 7 & 14 & 31 & 74 & 175 & 436 & 1154 & 3078 & 8343 & 23431 \\
\hline hyp & - & - & - & - & - & - & - & - & - & 4 & 25 & 120 & 459
\end{tabular}

Table 4: Numbers of prime closed manifolds up to complexity 12

\begin{tabular}{r||c|c|c|c|c|c|c} 
complexity & 1 & 2 & 3 & 4 & 5 & 6 & 7 \\
\hline \hline one cusp & - & 2 & 9 & 52 & 223 & 913 & 3388 \\
\hline two cusps & - & - & - & 4 & 11 & 48 & 162 \\
\hline three cusps & - & - & - & - & - & 1 & 2
\end{tabular}

Table 5: Numbers of hyperbolic cusped manifolds up to complexity 7

boundary. We will not review this notion here, but we mention it is implied by hyperbolicity. Using this fact, two groups of people investigated hyperbolic 3-manifolds of low complexity. Callahan-Hildebrandt-Weeks [3], using SnapPea [30, classified cusped manifolds up to complexity 7, getting the figures of Table 5. (A census exists also for non-orientable cusped hyperbolic manifolds, and it contains much fewer elements).

Frigerio-Martelli-Petronio [7] (see also [5, 6]) more recently turned their attention to hyperbolic manifolds with non-empty geodesic boundary, and found the numbers shown in Table 6 ,

\begin{tabular}{r||c|c|c} 
complexity & 2 & 3 & 4 \\
\hline \hline$\Sigma_{2}$ & 8 & 76 & 628 \\
\hline$\Sigma_{2} \cup T$ & - & 1 & 18 \\
\hline$\Sigma_{2} \cup T \cup T$ & - & - & 1 \\
\hline$\Sigma_{3}$ & - & 74 & 2034 \\
\hline$\Sigma_{3} \cup T$ & - & - & 12 \\
\hline$\Sigma_{4}$ & - & - & 2340
\end{tabular}

Table 6: Numbers of hyperbolic manifolds with compact and non-empty geodesic boundary up to complexity 4 , subdivided according to the type of $\partial \bar{M}$ 


\begin{tabular}{c||c|c|c||c} 
boundary type & $\#$ (gluings) & hyperbolic & non-hyperbolic & total \\
\hline \hline$\emptyset$ & 37 & - & 17 & 17 \\
\hline$T$ & 81 & 9 & 21 & 30 \\
\hline$T \sqcup T$ & 9 & 2 & 5 & 7 \\
\hline$\Sigma_{2}$ & 113 & 63 & 16 & 79 \\
\hline$\Sigma_{2} \sqcup T$ & 2 & 2 & - & 2 \\
\hline$\Sigma_{3}$ & 56 & 56 & - & 56 \\
\hline \hline Total & 298 & 132 & 59 & 191
\end{tabular}

Table 7: Numbers of distinct manifolds arising from orientation-preserving gluings of the faces of a truncated octahedron, with boundary spheres capped off

\section{Appendix (by Damian Heard and Ekaterina Pervova): The complete answer}

The complete answer to Question 0.1 (actually, to its equivalent formulation Question (3.4) is contained in Table 7. The hyperbolic entries of this table have been discussed in the body of the paper (note that 63 arises as the sum of $14+8+4$ from Proposition 5.2 plus the 37 from Proposition [5.3). They were all obtained using the software "Orb" 9] (or confirmed by "Orb" when they were known from [7]). Using randomization of triangulations, "Orb" also grouped together homeomorphic manifolds it could not construct a hyperbolic structure for, finding precisely the numbers shown in the table. To show that the numbers were indeed the correct ones we were then left with the following tasks:

1. Prove that among the groups of apparently non-hyperbolic manifolds produced by "Orb" there were no duplicates;

2. Prove that the apparently non-hyperbolic manifolds indeed were nonhyperbolic.

The "Recognizer" The basic tool we have used to achieve both these tasks is the "3-Manifold Recognizer," a software written by Tarkaev and Matveev [20]. The input to this program is a triangulation of a 3-manifold $M$ (so we had to subdivide our octahedron, and actually switch to the dual 
viewpoint of spines) and its output is the "name" of $M$, by which we mean the following:

- For a Seifert $M$, (one of) its Seifert structure(s);

- For a hyperbolic $M$, its presentation(s) as a Dehn filling of a manifold in the tables of Weeks [3];

- For a prime $M$ having JSJ decomposition into more than block, the name (as just illustrated) of the blocks, together with the gluing instructions between the blocks;

- For a non-prime manifold, the names (as just illustrated) of its prime summands.

The program is not guaranteed to always find the name of the manifold (for instance, it does not even attempt to do this for manifolds with boundary of genus 2 or more, and it happens to fail also in other cases). But it can always compute the first homology and, in the case of boundary of genus at most 1, the Turaev-Viro invariants [28, which turned out to be very useful for us.

Closed manifolds For the 37 gluing patterns in Table 3 defining closed manifolds, task 2 (proof of non-hyperbolicity) was not an issue, since it has been known for a long time [18] that closed hyperbolic manifolds start appearing in complexity 9, whereas a gluing of the octahedron has complexity at most 4. For task 1 (proof that the grouping by "Orb" contains no duplicates), we have run the "Recognizer," that successfully identified all the manifolds (this was also independently done by Tarkaev). From the names (all manifolds turned out to be Seifert or connected sums of Seifert) we could see there were either 16 or 17 of different ones. The only uncertainty was related to the fact that two manifolds were recognized to be the connected sum of two copies of the lens space $L(3,1)$, the point being that $L(3,1)$ has no orientation-reversing automorphism. So, even if one looks at orientable but non-oriented manifolds, there are two distinct ways of performing the connected sum of $L(3,1)$ with itself.

We then had to examine the two triangulations by hand, introducing an arbitrary orientation on each of them. For each triangulation we then found the non-trivial sphere realizing the connected sum. Cutting along this sphere 
and capping off, we saw that in one case the two connected summands were distinctly oriented copies of $L(3,1)$, while in the other case we got two copies of $L(3,1)$ with the same orientation. Thus, the manifolds were respectively $L(3,1) \#(-L(3,1))$ and $L(3,1) \# L(3,1)$, hence distinct.

One-cusped manifolds For the 81 triangulations from Table 3 giving manifolds bounded by one torus we followed approximately the same strategy. After the work with "Orb" already described above (recognition of hyperbolic manifolds and grouping of the others), we had 21 manifolds $\left\{M_{i}\right\}_{i=1}^{21}$ we should realize tasks 1 and 2 on. We proceeded as follows:

1. To carry out task 1 , i.e. to prove that there were no duplicates among the 21 manifolds, we again employed the "Recognizer", using which we calculated their first homology groups and Turaev-Viro invariants up to order 16 . From this computation we deduced that $M_{i} \neq M_{j}$ for $1 \leqslant i<j \leqslant 21$ except possibly for $i=1,2,3,4$ and $j=i+4$. For the four pairs of manifolds left, we showed the homeomorphism was impossible by analyzing the JSJ decompositions. Specifically, $M_{1}$ and $M_{5}$ turned out to be Seifert and distinct, and the same happened for $M_{2}$ and $M_{6}$, whereas $M_{3}$ and $M_{7}$ had non-trivial JSJ decompositions, with the same blocks but different gluing matrices, and analogously for $M_{4}$ and $M_{8}$.

2. In order to complete task 2 , i.e. to show that the manifolds were not hyperbolic, we computed the JSJ decomposition also for $M_{9}, \ldots, M_{21}$ (again using the "Recognizer"). In all cases we obtained a decomposition (sometimes trivial) consisting of Seifert pieces. (In one case the "Recognizer" failed to return the answer right away, but we were able to transform the triangulation by hand into one that the "Recognizer" could handle).

Two-cusped manifolds "Orb" reduced the issue of counting these manifolds to that of realizing tasks 1 and 2 on 5 of them, which was easy in this case using the "Recognizer":

1. For task 1, we calculated the Turaev-Viro invariants of the manifolds, which was sufficient to prove that all 5 manifolds were indeed distinct; 
2. For task 2, we found the JSJ decomposition of the manifolds. It turned out that either this decomposition was non-trivial or the manifold in question was Seifert. Hence indeed none of the manifolds was hyperbolic.

Genus-2 boundary For the 113 gluing patterns from Table 3 giving manifolds bounded by one genus- 2 surface, "Orb" showed there were 16 apparently non-hyperbolic and apparently distinct manifolds, and also provided a presentation for the fundamental group of each of them. These 16 manifolds were then treated as follows:

1. To address task 1, we computed the first homology of the manifolds (with the "Recognizer") and the Turaev-Viro invariants (by hand). Using these invariants, we were able to break down the set of 16 manifolds into some pairs and some 4-tuples of potentially equal manifolds, and also to single out two individual manifolds which were distinct from all the other ones. For the resulting groups of manifolds we then used the presentations of their fundamental groups to calculate the homology of the 3-fold coverings, which allowed us to distinguish almost all manifolds in these groups. This left only two pairs of manifolds, to which the same technique could not apply because the fundamental groups were isomorphic. To tackle these, we found, by hand, their JSJ decompositions and examined the gluings. In both cases the decomposition consisted of a genus-2 handlebody and a solid torus glued along an annulus. In one case the annuli used in the gluing were the same but the two gluings were along non-isotopic homeomorphisms. In the second case the annuli in the boundaries of the respective handlebodies were different, i.e. not related by any homeomorphism of the handlebodies.

2. To realize task 2, we showed that each of the 16 manifolds contained an essential annulus (which was explicitly constructed). For this, we employed the dual viewpoint of so-called simple spines, constructing for each of the manifolds in question a simple spine with an annulus component or a Möbius strip component. We then checked that in each case the annulus dual to the core of the annulus component or that dual to the boundary of the Möbius strip component, whichever was relevant, was actually essential in the manifold. 


\section{References}

[1] W. Klingenberg, "Riemannian Geometry," De Gruyter Std. in Math. Vol. 1, Berlin-New York, 1982.

[2] R. Benedetti - C. Petronio, "Lectures on Hyperbolic Geometry," Springer-Verlag, Berlin-Heidelberg-New York, 1992.

[3] P. J. Callahan - M. V. Hildebrandt - J. R. Weeks, A census of cusped hyperbolic 3-manifolds. With microfiche supplement, Math. Comp. 68 (1999), 321-332.

[4] D. B. A. Epstein, R. C. Penner, Euclidean decomposition of noncompact hyperbolic manifolds, J. Differential Geom. (1) 27 (1988), 67-80.

[5] R. Frigerio - B. Martelli - C. Petronio, Complexity and Heegaard genus of an infinite class of compact 3-manifolds, Pacific J. Math. 210 (2003), 283-297.

[6] R. Frigerio - B. Martelli - C. Petronio, Dehn filling of cusped hyperbolic 3-manifolds with geodesic boundary, J. Differential Geom. 64 (2003), 425-455.

[7] R. Frigerio - B. Martelli - C. Petronio, Small hyperbolic 3manifolds with geodesic boundary, Exp. Math. 13 (2004), 171-184.

[8] R. Frigerio - C. Petronio, Construction and recognition of hyperbolic 3-manifolds with geodesic boundary, Trans. Amer. Math. Soc. 356 (2004), 3243-3282.

[9] D. Heard, "Orb", The computer program for finding hyperbolic structures on hyperbolic 3-orbifolds and 3-manifolds, available from http://www.ms.unimelb.edu.au/ snap/orb.html

[10] J. Hempel, "3-Manifolds," Ann. of Math. Studies, 86, Princeton, (1976).

[11] S. KoJima, Polyhedral decomposition of hyperbolic manifolds with boundary, Proc. Work. Pure Math. 10 (1990), 37-57. 
[12] S. KoJima, Polyhedral decomposition of hyperbolic 3-manifolds with totally geodesic boundary, In: "Aspects of low-dimensional manifolds, Kinokuniya, Tokyo", Adv. Stud. Pure Math. 20 (1992), 93-112.

[13] B. Martelli, Complexity of 3-manifolds, In: "Spaces of Kleinian groups", London Math. Soc. Lec. Notes Ser. 329 (2006), 91-120.

[14] B. Martelli, C. Petronio, 3-manifolds having complexity at most 9, Exp. Math. 10 (2001), 207-237.

[15] B. Martelli, C. Petronio, A new decomposition theorem for 3manifolds, Illinois J. Math. 46 (2002), 755-780.

[16] S. V. MATVEev, The complexity of three-dimensional manifolds and their enumeration in the order of increasing complexity, Soviet Math. Dokl. 38 (1989), 75-78.

[17] S. V. Matveev, Complexity theory of three-dimensional manifolds, Acta Appl. Math. 19 (1990), 101-130.

[18] S. V. MATVEev, Algoritghmic topology and classification of 3-manifolds, ACM-monographs Vol. 9, Springer-Verlag, BerlinHeidelberg-New York, 2003.

[19] S. V. Matveev, Recognition and tabulation of 3-manifolds, Dokl. Akad. Nauk 400 (2005), 26-28 (Russian).

[20] S. V. Matveev - V. V. TARkaev, "Three-manifold Recognizer", a computer program for recognition of 3-manifolds, available from http://www.csu.ac.ru/ trk/spine/.

[21] G. Perelman, The entropy formula for the Ricci flow and its geometric applications, preprint math.DG/0211159.

[22] G. Perelman, Ricci flow with surgery on three-manifolds, preprint math.DG/0303109.

[23] G. Perelman, Finite extinction time for the solutions to the Ricci flow on certain three-manifolds, preprint math.DG/0307245.

[24] J. G. Ratcliffe, "Foundations of Hyperbolic Manifolds," Second Edition, Graduate Texts in Math. 149, Springer-Verlag, New York, 2006. 
[25] M. Sakuma - J. R. Weeks, The generalized tilt formula, Geom. Dedicata 50 (1995), 1-9.

[26] M. Scharlemann, Heegaard splittings of compact 3-manifolds, In: "Handbook of geometric topology" (R. Daverman and R. Sherr, eds.), North-Holland, Amsterdam (2002), 921-953.

[27] W. P. Thurston, "The Geometry and Topology of 3-manifolds," mimeographed notes, Princeton, 1979.

[28] V. Turaev - O. Viro, State sum invariants of 3-manifolds and quantum 6j-symbols, Topology (4) 31 (1992), 865-902.

[29] A. UshiJima, The tilt formula for generalized simplices in hyperbolic space, Discrete Comput. Geom. 28 (2002), 19-27.

[30] J. R. WeEks, "SnapPea", The hyperbolic structures computer program, available from www.geometrygames.org.

Dipartimento di Matematica Applicata

Via Filippo Buonarroti, 1C

56127 PISA - Italy

petronio@dm.unipi.it

RedTribe

Carlton, Victoria

Australia 3053

damianh@redtribe.com

Dipartimento di Matematica Applicata

Via Filippo Buonarroti, 1C

56127 PISA - Italy

pervovalcsu.ru 